\title{
Impedance Analysis of Colloidal Gold Nanoparticles in Chromatography Paper for Quantitation of an Immunochromatographic Assay
}

\author{
Fumitaka HorI, ${ }^{\dagger}$ Yuji HARAdA, Tatsumi KuretaKe, and Shigeyasu UnO ${ }^{\dagger}$ \\ Department of Electrical Systems, Graduate School of Science and Engineering, Ritsumeikan University, \\ 1-1-1 Nojihigashi, Kusatsu, Shiga 525-8577, Japan
}

\begin{abstract}
A detection method of gold nanoparticles in chromatography paper has been developed for a simple, cost-effective and reliable quantitation of immunochromatographic strip test. The time courses of the solution resistance in chromatography paper with the gold nanoparticles solution are electrochemically measured by chrono-impedimetry. The dependence of the solution resistance on the concentration of gold nanoparticles has been successfully observed. The main factor to increase the solution resistance may be obstruction of the ion transport due to the presence of gold nanoparticles. The existence of gold nanoparticles with $1.92 \times 10^{9}$ particles $/ \mathrm{mL}$ in an indistinctly-colored chromatography paper is also identified by a solution resistance measurement. This indicates that the solution resistance assay has the potential to lower the detection limit of the conventional qualitative assay.
\end{abstract}

Keywords Immunochromatography, chromatography paper, gold nanoparticles, solution resistance

(Received December 1, 2015; Accepted December 7, 2015; Published March 10, 2016)

\section{Introduction}

In recent years, chromatography paper (ChrPr) and gold nanoparticles (AuNPs) have attracted much attention in the biosensor field. Biosensors with ChrPr provide advantages in low cost and disposability. ${ }^{1-4}$ Biosensors with AuNPs also provide some advantages in helping electron transport or mediation on the sensor electrodes, ${ }^{5}$ and a surface plasmon resonance effect used for cancer detection. ${ }^{6,7}$ One of the examples of the bioassay exploiting ChrPr and AuNPs is the immunochromatographic strip test (IST), which is a simple and rapid flowing assay ${ }^{8}$ used for tests of pregnancy, ${ }^{9}$ influenza ${ }^{10}$ and cancer tumor. ${ }^{11}$ In IST, the commonly used labels are AuNPs. ${ }^{12-14}$ IST is generally a qualitative method based on a color line development, as described in Fig. 1. However, if there is a small number of the target analytes within the liquid sample, the AuNPs agglutination will be weak. ${ }^{15}$ This weak agglutination leads to a low concentration of AuNPs, and eventually causes a weakly-colored detection line, which might be invisible for the operator. ${ }^{16}$ Consequently, the assay has a possibility to be evaluated as a negative result, called a "false negative result". ${ }^{8}$ To avoid this, various quantitative methods have been investigated. In one approach, a method to measure the light intensity on the detection area is used. ${ }^{17-19}$ However, it sometimes requires some complex and expensive instrumentations. ${ }^{18}$ In another approach, a magnetic nano-gold microsphere $(\mathrm{MnGM})$ is exploited as a core of labels for better

† To whom correspondence should be addressed.

E-mail: ro0016xf@ed.ritsumei.ac.jp (F. H.); suno@fc.ritsumei. ac.jp (S. U.) detection of label agglutination..$^{20,21}$ In this method, a target analyte captured with MnGM would have more labels than the general IST. It achieves to obtain a very low probability of false negative results, but it is not cost-effective due to the complexity in the synthesis of MnGM. ${ }^{20}$ As the electrochemical approaches, chronoamperometry (CA) and electrochemical impedance spectroscopy (EIS) are used. CA and EIS are the general electrochemical approaches, and applied in the various research areas.,22-26 CA and EIS are especially adequate for the latest research related to the electrical characteristics of unique paper. ${ }^{3,27-29}$ The latest IST research is no exception to the use of CA and EIS. The measurement principle of the quantitative IST using $\mathrm{CA}$ is an oxidation-reduction reaction occurring on the

(a)

(b)

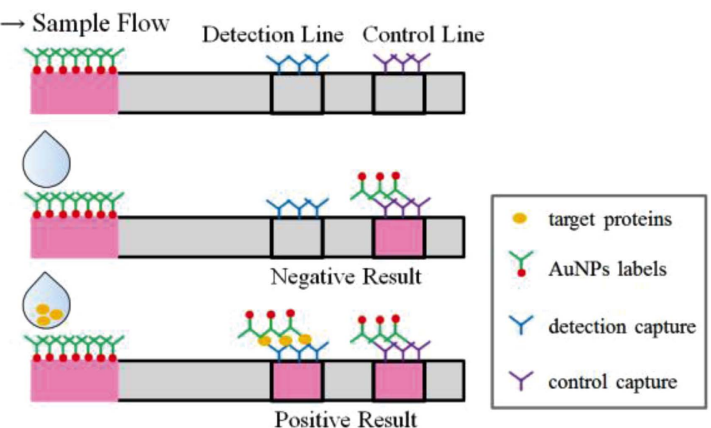

Fig. 1 Schematic illustrations of the principle of the IST. (a) Before detection. (b) Negative result obtained for liquid sample without target analytes. (c) Positive result obtained for a liquid sample with target analytes. 


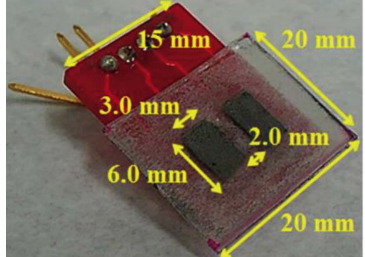

(a)

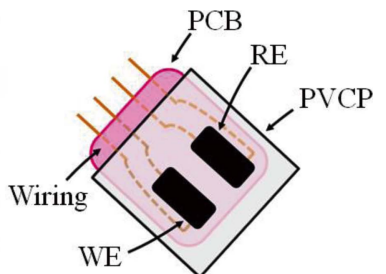

(b)
Fig. 2 Electrochemical PCBE chip used in this experiments. (a) Photograph of the top view of the PCBE chip. (b) Schematic description of the PCBE chip.

modified electrodes underneath the detection area. ${ }^{30,31}$ The measurement principle using EIS is to measure the capacitance on a color line using the modified electrodes with polymer layers. ${ }^{32,33}$ However, both methods using CA and EIS are costly because they need additional factors such as enzymes, mediators or polymers in addition to electrodes. On the other hand, a study to quantitate IST only with electrodes has been reported. ${ }^{34}$ This study focuses on latex beads (LBs) as one widely used label, and measures solution resistance $\left(R_{\text {sol }}\right)$ on the detection area in the presence and absence of LBs at a frequency where $R_{\text {sol }}$ dominates the total impedance. This might be the most appropriate quantitation method, since it can be applied to the conventional IST kits without any amendment. To apply this method to the practical IST, it should be confirmed that it is also feasible for other general labels, such as AuNPs and liposomes. ${ }^{35}$ The feasibility should be verified experimentally because the particle diameter of generally-used AuNPs (of the order of tens of nanometer) ${ }^{36}$ is smaller than that of LBs (several micrometer). ${ }^{34}$ In addition, it should be also confirmed that the lower limit of the $R_{\text {sol }}$ assay is below that of eye detection.

In this paper, we describe the chrono-impedimetry (CI) method used to detect AuNPs by measuring $R_{\text {sol }}$ at a fixed frequency. We confirmed that this method enables one to distinguish the different AuNPs concentrations in the sample solutions dropped onto $\mathrm{ChrPr}$ if the color of $\mathrm{ChrPr}$ is not recognized. In addition, we propose a detailed mechanism of the increase in $R_{\text {sol }}$ caused by AuNPs and an application to the practical IST. This would lead to a simpler, more cost-effective and reliable quantitation method of IST.

\section{Experimental}

\section{Reagents and materials}

AuNPs suspension (CAT No. 753688) containing $100 \mathrm{~nm}$ diameter AuNPs whose concentration is $3.84 \times 10^{9}$ particles $/ \mathrm{mL}$ in $0.1 \mathrm{mM}$ PBS of pH 5.0 was purchased from Sigma-Aldrich. It was stored at $4^{\circ} \mathrm{C}$ in the refrigerator. ChrPr (CAT No. 3001861) was purchased from Whatman, and stored at room temperature in the desiccator.

\section{Electrochemical electrode chip design}

A print circuit board (PCB), which is usually used in circuit implementation, and a polyvinyl chloride plate (PVCP) were used to fabricate a print circuit board electrode (PCBE), as shown in Fig. 2. Since PCB was originally designed as a fourterminal electrode, it has four wirings. However, these wirings are paired and common to utilize PCBE as a two-terminal electrode in this study. PCBE has two terminals: a working electrode (WE) and a reference electrode (RE). Carbon paste

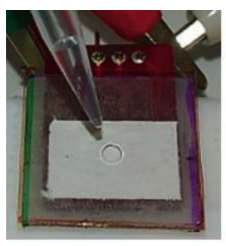

(a)

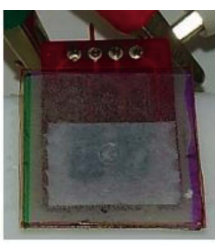

(b)

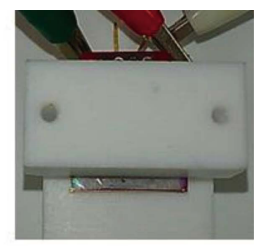

(c)
Fig. 3 Photograph of the arrangement of measurements. (a) Before dropping AuNPs solution onto ChrPr on PCBE into the hole on PVCP. (b) $\mathrm{ChrPr}$ covered with PVCP after flowing AuNPs solution. (c) PCBE, ChrPr and PVCP in (b) pressed and held by a weight.

(CAT No. 001010, BAS) was used for WE and RE. The first step to fabricate PCBE is to bond PVCP $(20 \mathrm{~mm} \times 20 \mathrm{~mm}$, $0.5 \mathrm{~mm}$ thickness) to PCB by a double-sided tape. PVCP has two rectangular holes $(3 \mathrm{~mm} \times 6 \mathrm{~mm}, 2 \mathrm{~mm}$ distance $)$ to be filled with carbon paste. After pressing carbon paste into the holes, the PCBE fabrication process is terminated in polishing the surface of the carbon electrodes by filter paper. The surface of the electrodes should be flat to make sure that $\mathrm{ChrPr}$ uniformly touches the surface of PCBE.

\section{Impedance measurement}

EIS and CI were performed in this study. EIS is a popular method used to measure the dependence of the impedance $(Z)$ on the frequency $(f)$ of the AC voltage applied between WE and RE. The $f$ where $R_{\text {sol }}$ appears is identified by EIS. On the other hand, CI is a method used to measure the time course of $Z$ at a fixed $f$. When $f$ is carefully chosen to capture $R_{\text {sol }}$, the time course of $R_{\text {sol }}$ is given by CI. In the case of EIS, $Z$ was measured 12 points per one order of magnitude in $f$ at zero DC bias potential with $\mathrm{AC}$ amplitude of $5 \mathrm{mV}$. In the CI measurement, $Z$ was measured per $2 \mathrm{~s}$ at zero DC bias potential with $\mathrm{AC}$ amplitude of $5 \mathrm{mV}$. During the CI measurements, $f$ was fixed to $5625 \mathrm{~Hz}$ because it is the most appropriate frequency to measure $R_{\text {sol }}$ in this study, as mentioned in the Results section.

\section{Measurement procedure}

A $10-\mathrm{mL}$ portion of an AuNPs solution with $3.84 \times 10^{9} \mathrm{~mL}^{-1}$ was centrifuged at $2500 \mathrm{rpm}$ for $2 \mathrm{~h}$ by a Centrifuge C-12B (CAT No. 1-3126-21, AS ONE), and separated into two parts. One was $0.5 \mathrm{~mL}$ with a high AuNPs concentration $(7.68 \times$ $\left.10^{10} \mathrm{~mL}^{-1}\right)$; the other was $9.5 \mathrm{~mL}$ without any AuNPs $\left(0 \mathrm{~mL}^{-1}\right)$. The other AuNPs solutions with concentrations of $3.84 \times$ $10^{8} \mathrm{~mL}^{-1}, 1.92 \times 10^{9} \mathrm{~mL}^{-1}, 3.84 \times 10^{9} \mathrm{~mL}^{-1}$ and $3.84 \times 10^{10} \mathrm{~mL}^{-1}$ were prepared from those solutions. During the experiment, except in the sampling step, all of AuNPs solutions were stored at $4{ }^{\circ} \mathrm{C}$ in a refrigerator so as to avoid any agglutination of the AuNPs. At the beginning of the measurement, $15 \mathrm{~mm} \times 10 \mathrm{~mm}$ of ChrPr was set on PCBE so as to ensure the ChrPr arrangement. In addition, another PVCP $(20 \mathrm{~mm} \times 20 \mathrm{~mm}, 0.1 \mathrm{~mm}$ thickness $)$ with a hole of $3 \mathrm{~mm}$ in diameter was set on the ChrPr to fix the liquid casting position. For a good arrangement, the size of PVCP should be the same as the PCBE. After the PVCP setting, a $30-\mu \mathrm{L}$ portion of the AuNPs solution was dropped into the hole on the PVCP, as shown in Fig. 3(a), and thereafter, the upper PVCP was removed. If there are some air bubbles between PCBE and ChrPr after the dropping, ChrPr is slid so as to release the air, since they can be a source of serious fluctuation in the experimental result. After completion of flowing the AuNPs solution into ChrPr, a new PVCP $(20 \mathrm{~mm} \times 20 \mathrm{~mm}$, 
(a)

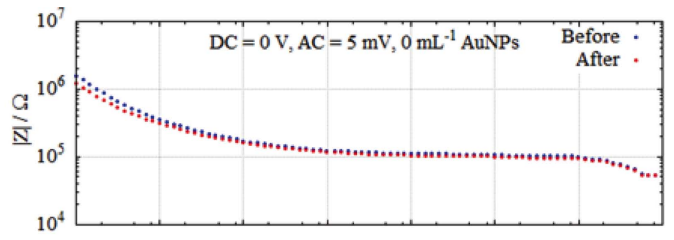

(b)
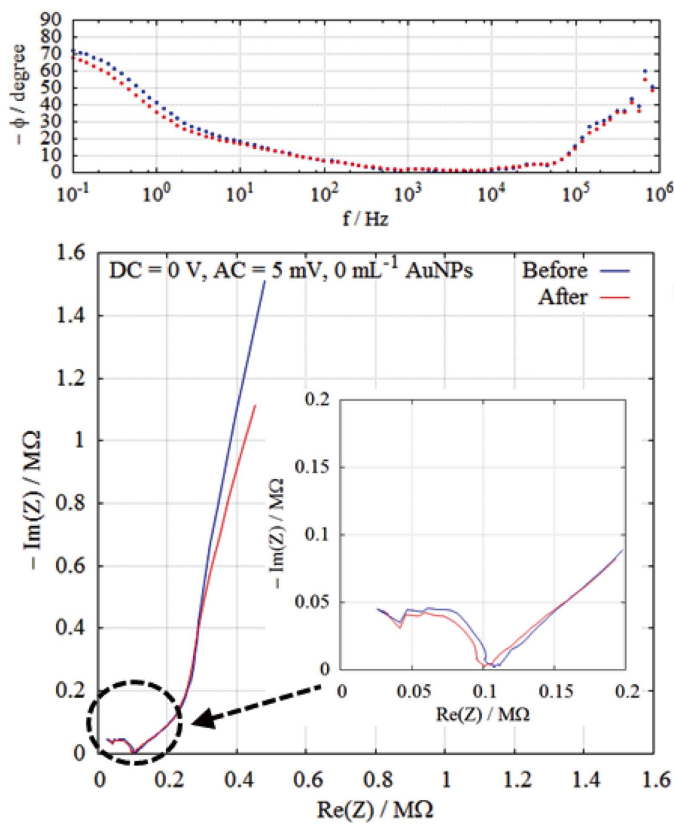

Fig. 4 Representative results of the EIS measurement with $0 \mathrm{~mL}^{-1}$ AuNPs dropped onto $\mathrm{ChrPr}$ on PCBE before and after the $\mathrm{CI}$ measurements. (a) Bode diagram. (b) Nyquist diagram. Inset is the enlarged view of (b) up to $0.2 \mathrm{M} \Omega$

$0.1 \mathrm{~mm}$ thickness) was set on $\mathrm{ChrPr}$ so as to prevent water from evaporation, as shown in Fig. 3(b). Furthermore, as shown in Fig. 3(c), PCBE, ChrPr and the PVCP were pressed and held by a weight $(15.38 \mathrm{~g})$. All of the electrochemical measurements were performed with ALS/CH Instruments Electrochemical Analyzer Model 6081E (CAT No. 013046, BAS) at room temperature. The humidity was controlled at around 60 percent by the humidifier placed in the laboratory. Prior to each measurement, the upper PVCP and the PCBE were washed with $0 \mathrm{~mL}^{-1}$ AuNPs. The next measurement was performed after the washing procedure. The upper PVCP was renewed for every AuNPs concentration.

\section{Results and Discussion}

\section{Results}

Figure 4 shows representative results of the EIS measurement with $0 \mathrm{~mL}^{-1}$ AuNPs dropped onto ChrPr on PCBE before and after the $\mathrm{CI}$ measurements. In Fig. 4(a), the vertical axes are $|Z|$ and the phase shift, and the horizontal axis is $f$. In the graph, electric double-layer capacitance is exhibited at a low- $f$ region (approximately $f<10^{3} \mathrm{~Hz}$ ), since the phase shift is nearly $-90^{\circ}$, and the parasitic capacitance due to the external wiring appears in the high- $f$ range (approximately $10^{5} \mathrm{~Hz}<f$ ). ${ }^{37}$ The curves in Fig. 4(a) in the range are similar to those seen in the EIS measurement while disconnecting PCBE with the electrochemical analyzer. It indicates that $Z$ in such a high- $f$ range is showing the parasitic capacitance. $R_{\text {sol }}$ can be observed

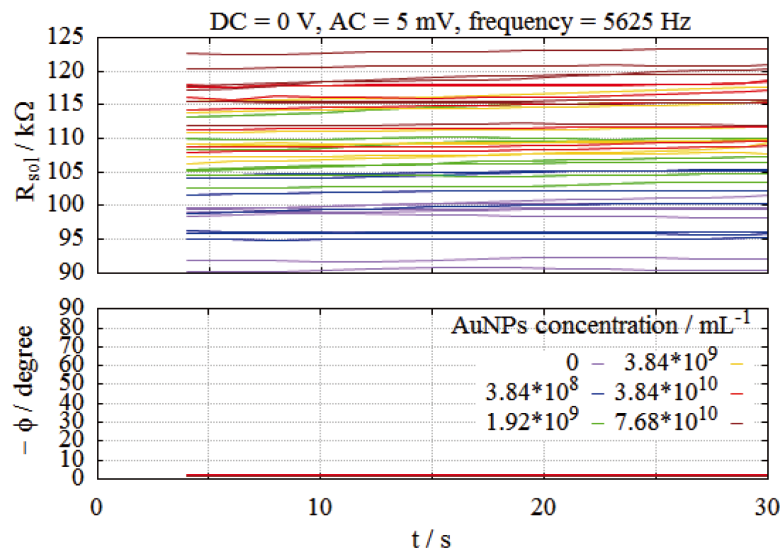

Fig. 5 The time courses of $R_{\text {sol }}$ and phase shift at $f=5625 \mathrm{~Hz}$ for various AuNPs concentrations (seven times each).

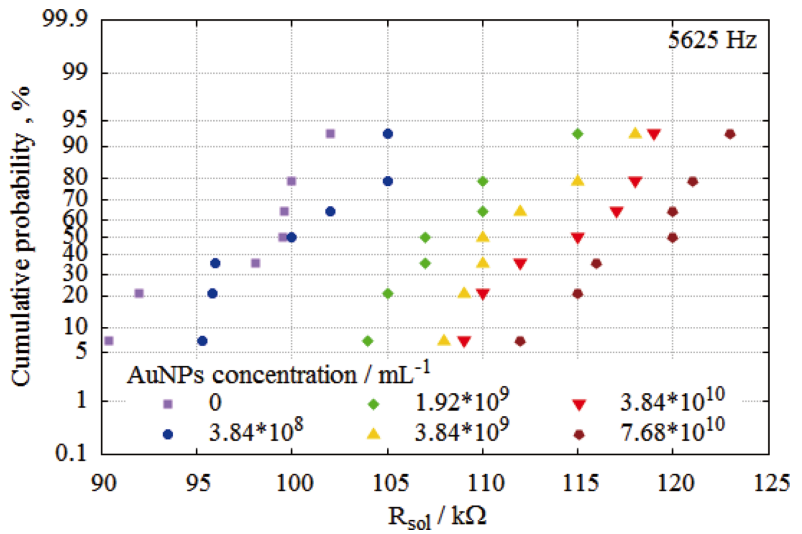

Fig. 6 Normal probability plot of $R_{\text {sol }}$ shown in Fig. 5 at $t=30 \mathrm{~s}$ for various AuNPs concentrations. Cumulative probability $(P)$ was calculated from $P=100 \times(j-0.5) / n$, where $n=7$ in each AuNPs concentration, and $j$ is each data number arranged in ascending order at every AuNPs concentration.

in the range of approximately $10^{3}-10^{5} \mathrm{~Hz}$, since the phase shift is close to $0^{\circ} .^{37}$ Note that the $f$ range where $R_{\text {sol }}$ appears does not change before and after the CI measurements. Therefore, a fixed $f$ will be sufficient to capture $R_{\text {sol }}$ during the CI measurements. In this work, $f=5625 \mathrm{~Hz}$ was chosen because the phase shift is the closest to $0^{\circ}$ there. Next, the time courses of $R_{\text {sol }}$ and the phase shift at $5625 \mathrm{~Hz}$ for various AuNPs concentrations were measured for $30 \mathrm{~s}$ using CI, as shown in Fig. 5. The vertical axes are $R_{\text {sol }}$ and the phase shift. Horizontal axis is time. The graph indicates that $Z$ is equal to $R_{\text {sol }}$ because all of these phase shifts were stable and close to $0^{\circ}$ during the measurements. In Fig. 5, the rate of change in $R_{\text {sol }}$ is diminishing as time passes. As a result, $R_{\text {sol }}$ becomes stable at the end of each measurement. A normal probability plot of $R_{\text {sol }}$ at $30 \mathrm{~s}$ is shown in Fig. 6. Figure 7 shows the $R_{\text {sol }}$ dependence on AuNPs concentration given by $R_{\text {sol }}$ in Fig. 6 . Note that $R_{\text {sol }}$ tends to increase due to AuNPs concentration in the liquid sample.

\section{Discussion}

The first discussion is to clarify the mechanism of the increase in $R_{\text {sol }}$ due to that of the AuNPs concentration. We start our discussion by expressing $R_{\text {sol }}$ as an equation.

In general, solution resistance is expressed by 


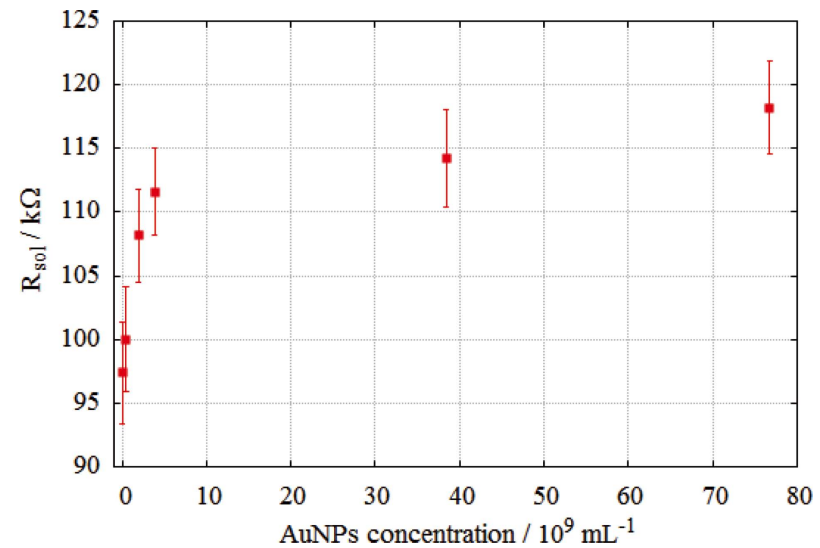

Fig. $7 R_{\text {sol }}$ dependence on the AuNPs concentration given by $R_{\text {sol }}$ in Fig. 6.

(a)

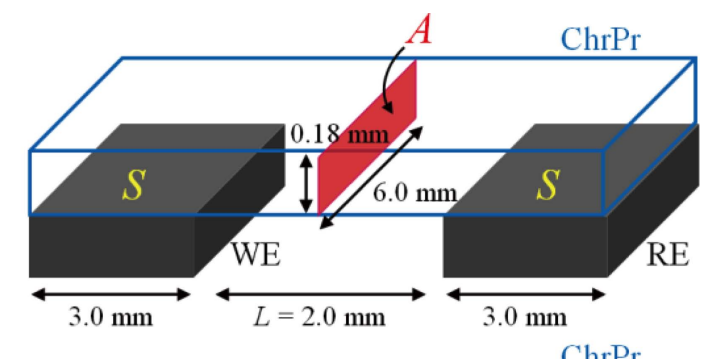

(b)

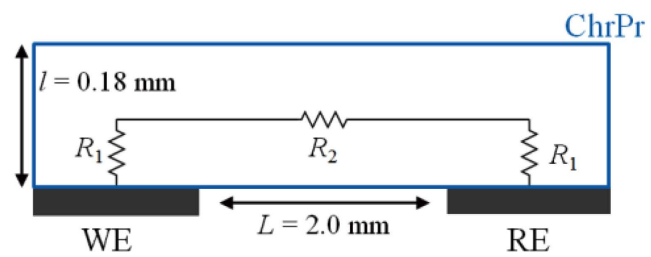

Fig. 8 Schematic diagram of ChrPr including solution on the electrodes: (a) geometry, (b) cross sectional view. The height of $\mathrm{ChrPr}$ is $l(0.18 \mathrm{~mm})$, and the distance between WE and RE is $L(2 \mathrm{~mm})$. $R_{1}$ is a solution resistance which is vertical to the electrode surface, and $R_{2}$ is a solution resistance which is horizontal to that. $S$ is the effective area of the electrode surface.

$$
R=\frac{1}{\kappa} \times \frac{L}{A},
$$

where $\kappa$ is solution conductivity $[\mathrm{S} / \mathrm{m}], L$ is distance between electrodes $[\mathrm{m}]$, and $A$ is cross-section area $\left[\mathrm{m}^{2}\right] .{ }^{38}$ Considering the geometry described in Fig. 8(a), the actual resistance between two electrodes underneath $\mathrm{ChrPr}$ can be modeled as shown in Fig. 8(b), where $R_{1}$ represents the resistance near the electrodes given by

$$
R_{1}=\frac{1}{\kappa} \times \frac{l / 2}{S}
$$

and $R_{2}$ is the solution resistance in the ChrPr between two electrodes given by

$$
R_{2}=\frac{1}{\kappa} \times \frac{L}{A}
$$

For simplicity, the length of $R_{1}$ is assumed to be half of the height of ChrPr, and that of $R_{2}$ is also assumed to be the distance between the edge of WE and RE. From the above equations,

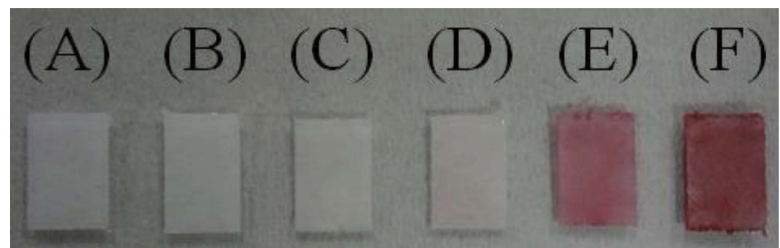

Fig. 9 Photograph of ChrPr with each concentration of AuNPs after dropped: (A) $0 \mathrm{~mL}^{-1}$, (B) $3.84 \times 10^{8} \mathrm{~mL}^{-1}$, (C) $1.92 \times 10^{9} \mathrm{~mL}^{-1}$, (D) $3.84 \times 10^{9} \mathrm{~mL}^{-1}$, (E) $3.84 \times 10^{10} \mathrm{~mL}^{-1}$, (F) $7.68 \times 10^{10} \mathrm{~mL}^{-1}$.

$R_{\text {sol }}$ can be expressed as

$$
R_{\mathrm{sol}}=2 R_{1}+R_{2}=\frac{1}{\kappa}\left(\frac{l}{S}+\frac{L}{A}\right) .
$$

From Eq. (4), two factors are responsible for increasing $R_{\text {sol }}$ by AuNPs. One is the decrease in $S$ due to the sediment of AuNPs on the electrode surface. Another is the decrease in $A$ due to the existence of AuNPs. The ion transport is obstructed by AuNPs filling some portions of the pores in the cellulose matrix of ChrPr. In this case, $\kappa, l$ and $L$ do not change. However, decrease in $S$ due to the sediment does not contribute to the increase in $R_{\text {sol }}$, because $l / S$ term in Eq. (4) is much smaller than $L / A$ for the given geometry $\left(l / S=0.01 \mathrm{~mm}^{-1}\right.$ and $\left.L / A=1.85 \mathrm{~mm}^{-1}\right)$. The main cause of the $R_{\text {sol }}$ increase is therefore due to the decrease in $A$. As the AuNPs concentration increases, the amount of AuNPs entering into cellulose matrix also increases. Therefore, it is appropriate to consider that $R_{\text {sol }}$ increases due to the AuNPs concentration.

In order to reduce the $R_{\text {sol }}$ variability at each AuNPs concentration in Fig. 7, consideration for its cause is required. One factor is the uncontrolled dispersion of AuNPs in ChrPr. Since the structure of the cellulose matrix of $\mathrm{ChrPr}$ is not completely uniform, the result of the transport and distribution of AuNPs would differ each time. In addition, there were no reagents to help AuNPs flow and diffuse in ChrPr in this study. Thus, there was no mechanism to spread the AuNPs in ChrPr uniformly. That affected the distribution of AuNPs in ChrPr, and it should become the factor of the fluctuation of $R_{\text {sol }}$. In the practical IST, the fluctuation range may decrease since the AuNPs dispersion is controlled by the capture antibody on a detection line. Other factors are the inaccurate handling at the PCBE fabrication and the human error in the measurement procedure. In a practical use, the preparation of the measurement will be automated and standardized.

The next discussion deals with the feasibility of our AuNPs detection for the application to IST. In a conventional IST kit, the maximum AuNPs concentration on a positive detection line depends on a category of the target analyte. In addition, a color reaction occurs invariably, and one method to qualify the result is eye detection. Figure 9 shows $\mathrm{ChrPr}$ soaked with liquid samples used in our experiment. The $\mathrm{ChrPr}(\mathrm{C})$ absorbing $1.92 \times 10^{9} \mathrm{~mL}^{-1}$ AuNPs may be regarded as the absence of AuNPs in eye detection. However the presence of AuNPs in that ChrPr was successfully identified by our electrochemical $R_{\text {sol }}$ measurement, as indicated in Fig. 7. Accordingly, our method could provide better sensitivity than the conventional eye detection. If that amount of AuNPs is agglutinated on a detection line in a practical IST kit, the presence of AuNPs may be detected by CI, but not by eyes. It indicates the potential to improve the detection limit of some conventional IST kits. That consequence increases the possibility of the $R_{\text {sol }}$ assay to avoid 
false negative results in the general IST.

The size of the target analytes also gives a positive suggestion to be able to detect target analytes with AuNPs in IST by our method. The diameters of the representative target analytes (the virus that infects humans, ${ }^{39-41}$ animals $^{42}$ or plants, ${ }^{43}$ and the allergic substance in foods ${ }^{44}$ ) have the same order of magnitude in size as AuNPs $(\sim 100 \mathrm{~nm})$. Thus, in IST, the agglutination caused by antigen-antibody reaction generates several times larger conjugates than AuNPs on a detection line. It causes more filling in the portions of the pores in the cellulose matrix of ChrPr than our basic research. Due to that, the ion transport would be more obstructed, and the $R_{\text {sol }}$ difference may become more pronounced. It indicates that there might be a great possibility to detect $R_{\text {sol }}$ remarkably in the conventional IST kits.

\section{Conclusions}

We have demonstrated the AuNPs detection in ChrPr by the CI method to develop a quantitative IST. We have successfully observed the increase in $R_{\text {sol }}$ due to the presence of AuNPs and the dependence of $R_{\text {sol }}$ on the AuNPs concentration. The increase in $R_{\text {sol }}$ has been attributed to an obstruction of ion transport due to the entrance of the AuNPs into the cellulose matrix of ChrPr. Our method would enable one to detect the presence of AuNPs in an immunochromatographic assay even when the coloring is weak enough to be regarded as false negative.

\section{Acknowledgements}

This work was supported by JSPS KAKENHI Grant Number 26289111

\section{References}

1. A. W. Martinez, S. T. Phillips, and G. M. Whitesides, PNAS, 2008, 105, 19606.

2. J. Yu, S. Wang, L. Ge, and S. Ge, Biosens. Bioelectron., 2011, 26, 3284.

3. R. F. Carvalhal, M. S. Kfouri, M. H. de O. Piazetta, A. L. Gobbi, and L. T. Kubota, Anal. Chem., 2010, 82, 1162.

4. A. W. Martinez, S. T. Phillips, M. J. Butte, and G. M. Whitesides, Angew. Chem. Int. Ed., 2007, 46, 1318.

5. R. F. Carvalhal, E. Carrilho, and L. T. Kubota, Bioanalysis, 2010, 2, 1663.

6. S. Eustis and M. A. El-Sayed, Chem. Soc. Rev., 2006, 35, 209.

7. I. H. El-Sayed, X. Huang, and M. A. El-Sayed, Nano Lett., 2005, 5, 829.

8. M. E. Koivunen and R. L. Krogsrud, LABMEDICINE, 2006, 37, 490.

9. M. J. Wheeler, "The Immunoassay Handbook", ed. D. Wild, 3rd ed., 2013, Chap. 22, Elsevier Science, Amsterdam.

10. Y. Tsuda, Y. Sakoda, S. Sakabe, T. Mochizuki, Y. Namba, and H. Kida, Microbiol. Immunol., 2007, 51, 903.

11. M. R. Suresh, "The Immunoassay Handbook", ed. D. Wild, 3rd ed., 2013, Chap. 56, Elsevier Science, Amsterdam.

12. M. Yamaguchi, S. Kosaka, Y. Tahara, and A. Date, ECS Transactions, 2008, 16, 67.

13. D. Zhang, P. Li, Y. Yang, W. Zhang, Z. Xiao, and X. Ding, Talanta, 2011, 85, 736.

14. R. H. Shyu, H. F. Shyu, H. W. Liu, and S. S. Tang, Toxicon, 2002, 40, 255.

15. E. Beck, J. Fan, K. Hendrickson, S. Kumar, R. Shively, W. Kramp, J. Villanueva, D. Jernigan, A. Klimov, L. M. Chen,
R. Donis, T. Williams, J. Pirkle, and J. Barr, Morbidity and Mortality Weekly Report, 2012, 61, 873.

16. B. O’Farrell, “Lateral Flow Immunoassay”, ed. R. Wong and H. Tse, 2010, Chap. 1, Humana Press, New York.

17. Q. Zeng, X. Mao, H. Xu, S. Wang, and G. Liu, Am. J. Biomed. Sci., 2009, 1, 70.

18. X. Mao, W. Wang, and T. E. Du, Sens. Actuators B, 2013, $186,315$.

19. Z. Li, Y. Wang, J. Wang, Z. Tang, J. G. Pounds, and Y. Lin, Anal. Chem., 2010, 82, 7008.

20. D. Tang, J. C. Sauceda, Z. Lin, S. Ott, E. Basova, I. Goryacheva, S. Biselli, J. Lin, R. Niessner, and D. Knopp, Biosens. Bioelectron., 2009, 25, 514.

21. J. Y. Liao and H. Li, Microchim. Acta, 2010, 171, 289.

22. S. Ito, N. L. C. Ha, G. Rothenberger, P. Liska, P. Comte, S. M. Zakeeruddin, P. Pechy, M. K. Nazeeruddin, and M. Gratzel, Chem. Commun., 2006, 38, 4004.

23. J. Drexler and C. Steinem, J. Phys. Chem. B, 2003, 107, 11245.

24. R. Gomez, R. Bashir, A. Sarikaya, M. R. Ladisch, J. Sturgis, J. P. Robinson, T. Geng, A. K. Bhunia, H. L. Apple, and S. Wereley, Biomedical Biodevices, 2001, 3, 201.

25. M. Ates, Progress in Organic Coatings, 2011, 71, 1.

26. A. H. Kyle, C. T. O. Chan, and A. I. Minchinton, Biophys. J., 1999, 76, 2640.

27. S. H. Ng, J. Wang, Z. P. Guo, J. Chen, G. X. Wang, and H. K. Liu, Electrochim. Acta, 2005, 51, 23.

28. H. P. Cong, X. C. Ren, P. Wang, and S. H. Yu, Energy Environ. Sci., 2013, 6, 1185.

29. C. Q. Peng, Y. S. Thio, and R. A. Gerhardt, Nanotechnology, 2008, 19, 505603.

30. K. Inoue, P. Ferrante, Y. Hirano, T. Yasukawa, H. Shiku, and T. Matsue, Talanta, 2007, 73, 886.

31. Y. Kiba, Y. Otani, T. Yasukawa, and F. Mizutani, Electrochim. Acta, 2012, 81, 14.

32. C. Fernandez-Sanchez, C. J. McNeil, and K. Rawson, Trends Anal. Chem., 2005, 24, 37.

33. C. Fernandez-Sanchez, C. J. McNeil, K. Rawson, and O. Nilsson, Anal. Chem., 2004, 76, 5649.

34. S. Iwahara, M. Miki, F. Hori, and S. Uno, Jpn. J. Appl. Phys., 2014, 53, 04EL07.

35. S. Shukla, H. Leem, and M. Kim, Anal. Bioanal. Chem., 2011, 401, 2581.

36. K. Faulstich, R. Gruler, M. Eberhand, D. Lentzsch, and K. Haberstroh, "Lateral Flow Immunoassay", ed. R. Wong and H. Tse, 2010, Chap. 9, Humana Press, New York.

37. I. D. Raistrick and D. R. Franceschetti, "Impedance Spectroscopy", ed. E. Barsoukov and J. R. Macdonald, 2nd ed., 2005, Chap. 2, John Wiley and Sons, New York.

38. A. J. Bard and L. R. Faulkner, "Electrochemical Methods Fundamentals and Applications", 2nd ed., 2001, Chap. 1, John Wiley and Sons, New York.

39. Y. Amano and Q. Cheng, Anal. Bioanal. Chem., 2005, 381, 156.

40. H. Ushijima, T. Fujimoto, W. E. G. Muller, and S. Hayakawa, Food Safety, 2014, 2, 37.

41. N. A. Afifi, A. M. El-Temawy, Gh. O. El-Sedafy, and S. H. Hanash, Egyptian Journal of Medical Microbiology, 2007, 16, 145.

42. S. Savic-Jevdenic, D. Trailovic, B. Vidic, and M. Jovanovic, Acta Veterinaria, 2006, 56, 515.

43. N. A. Byzova, I. V. Safenkova, S. N. Chirkov, V. G. Avdienko, A. N. Guseva, I. V. Mitrofanova, A. V. Zherdev, B. B. Dzantiev, and J. G. Atabekov, Biochemistry, 2010, 75, 1393.

44. S. Hideshima, S. Kuroiwa, M. Kimura, S. Cheng, and T. Osaka, Electrochim. Acta, 2013, 110, 146. 